\title{
STUDY ON SEAWATER INTRUSION AND INFLUENCING FACTORS BASED ON THE ADVECTION-DISPERSION EQUATION
}

\author{
ZHANG, L. ${ }^{1}-$ JIANG, Z. ${ }^{2 *}-$ WANG, H. $^{3}-$ QI, Q. ${ }^{1}-$ ZHU, Y. ${ }^{4}$

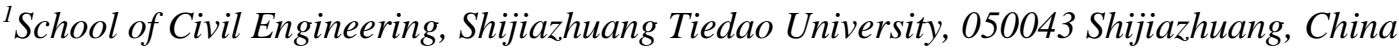 \\ ${ }^{2}$ China Railway Guangzhou Group Hainan Railway Co., Ltd., 570100 Haikou, China \\ ${ }^{3}$ Shijiazhuang City Water Conservancy and Hydropower Engineering Department, 050000 \\ Shijiazhuang, China \\ ${ }^{4}$ Hebei Institute of Water Science, 050000 Shijiazhuang, China \\ *Corresponding author \\ e-mail: 599787051@qq.com
}

(Received 21 $1^{\text {st }}$ May 2019; accepted $16^{\text {th }}$ Oct 2019)

\begin{abstract}
The purpose of this study was to explain the phenomenon of seawater intrusion (SWI) by numerical simulation and analysis. In this paper, a numerical method was introduced for solving the onedimensional advection-dispersion equation by the explicit finite difference method, and the corresponding convergence conditions and the boundary attenuation factor, $\beta$, were given, which can be convenient in studying the impact of different influencing factors on seawater intrusion. Additionally, the effects of six hydraulic factors on seawater intrusion were explored under steady state, namely, hydraulic conductivity, longitudinal dispersivity, hydraulic gradient, flux, evaporation and rainfall. Moreover, two different seawater intrusion types were also studied, namely active seawater intrusion and passive seawater intrusion. Meanwhile, the control variates method was adopted to explore the influence of a single factor on seawater invasion. According to the calculated results, with the increase of hydraulic conductivity, longitudinal dispersion, flux and evaporation, the degree of it will be aggravated, but the rainfall can play a role in mitigating seawater intrusion. Besides, under active seawater intrusion conditions, the growth of the numerical value of hydraulic gradient will aggravate seawater intrusion, while under passive seawater intrusion conditions, it will slow down the degree of invasion. All in all, the results and achievements are consistent with the actual situation, which can explain the phenomenon of seawater intrusion.
\end{abstract}

Keywords: seawater intrusion, numerical simulation, explicit finite difference method, active and passive seawater intrusion, seepage tank

\section{Introduction}

In coastal and island areas, seawater intrusion (SWI) has become one of the major geological hazards due to the over-exploitation of groundwater (Li et al., 1996). Seawater intrusion arising from aquifer depletion is often classified as active or passive, depending on whether seawater moves in the same direction as groundwater flow or not (Werner, 2017). Generally, hydrodynamic and hydrogeological conditions are essential for the formation of seawater intrusion (Xue et al., 1992). There are a variety of factors affecting seawater intrusion, which can also be divided into hydrodynamic factors and hydrogeological factors under natural conditions excluding human factors. Hydrodynamic factors include hydraulic gradient, flux, evaporation, rainfall, recharge and tidal action, while hydrogeological factors include hydraulic conductivity, longitudinal dispersivity, adsorption and porosity (Chen et al., 2013). According to the Technical regulations for monitoring and evaluating sea water intrusion issued by the 
State Oceanic Administration (Mao, 2014), the concentration of chloride ion at $250 \mathrm{mg} / \mathrm{L}$ can evaluate the degree of seawater intrusion.

Zhao et al. (2018) investigated the effects of some influencing factors on seawaterfreshwater mixing zones, namely, saturated hydraulic conductivity, porosity, molecular diffusivity, longitudinal and transverse dispersivity, by analyzing their uncertainty and sensitivity for the predicted seawater-freshwater mixing zones in simulation experiments. Moreover, based on the measured data of groundwater level and content in the transition zone, Zhang et al. (2015) and Zhen et al. (2015) found that there was a significant negative correlation between the seasonal variation of groundwater level and the seasonal variation of groundwater content. Then, Zhang (2015) determined that the correlation coefficient was above 0.92. Adel et al. (2014) found that salinization of fresh groundwater is highly associated with groundwater withdrawal based on historical hydraulic heads data. Qing et al. (2013) determined that rainfall and tidal effects have an important impact on seawater intrusion, and that rainfall can lower the degree of seawater intrusion. Liu et al. (2007) obtained that the increase of elevation difference between seawater and freshwater level will increase the speed of SWI. Zhang and Chen et al. (2015) proposed that local groundwater is subjected to anthropogenic activities and geochemical processes, such as evaporation, water-rock interaction, and ion exchange, thus affecting the degree of seawater intrusion. Emna et al. (2018) found local shallow groundwater is threatened by seawater intrusion because of the reduced rainfall and recharge capacity.

Based on the above research contents, six hydraulic factors were considered including hydraulic conductivity, longitudinal dispersivity, hydraulic gradient, flux, evaporation and rainfall in this paper. In order to study the influence of these six hydraulic factors on seawater intrusion and its final steady state, the control variates method was adopted in this paper. With other conditions unchanged, the influence of the change of one factor on the final steady state was considered separately. In this paper, the explicit finite difference method was adopted to solve the onedimensional advection-dispersion equation. The purpose of this method was to simulate the real salt migration phenomenon from the viewpoint of numerical calculation, analyze the mechanism of seawater intrusion, and explain the influence of various factors on the seawater intrusion under both steady state and two kinds of SWI types, active SWI and passive SWI (active seawater intrusion and passive seawater intrusion).

The one-dimensional advection-dispersion equation has been widely applied to describe the transportation process, which is utilized for the study of seawater intrusion (Yu, 2018). Compared with two-dimensional and three-dimensional models, it requires fewer parameters and remains as a simple and feasible method, and it is convenient to study the impact of the above six hydraulic factors on seawater intrusion. Nie et al. (2002) explained the problem of seawater intrusion through the advection-dispersion model of miscible fluid. Purnaditya et al. (2018) developed the mathematical model of groundwater flow and solute transport which is applicable to seawater intrusion mechanism.

With respect to solve one-dimensional advection-dispersion equation by finite difference method, Yu et al. (2018), from China, implemented Homotopy Analysis Method (HAM) to solve the one-dimensional advection-dispersion equation. Savovic et al. (2012), from Russia, solved one-dimensional advection- dispersion equation with variable coefficients by using explicit finite difference method and shown that explicit 
finite difference method is effective and accurate for solving the equation. Yang et al. (2016), from China, proposed a boundary value method for solving fractional order advection-dispersion equation. In the traditional numerical solution of the advectiondispersion equation, the longitudinal dispersivity coefficient is often regarded as a constant, but it is directly proportional to the size of the flow velocity. When the distribution of the flow velocity in space is not equal, neither the velocity nor the longitudinal dispersivity coefficient can be regarded as a constant. Comparing the methods described above, the methods presented in this paper combined the characteristics of seawater intrusion, considering active SWI and passive SWI, introducing the boundary attenuation factor, and giving the convergence conditions, which can simulate the real situation of seawater intrusion.

Therefore, the article aimed to study seawater intrusion through the introduction of a numerical method to solve the one-dimensional advection-dispersion equation using the finite difference method, and additionally investigated influencing factors, the effect strength of a single such factor, and seawater intrusion types.

\section{Research methods}

\section{One-dimensional advection-dispersion equation}

In the steady seepage field, if the change of water density caused by the change of solute concentration can be ignored by solving the advection-dispersion equation, the flow equation and the solute transport equation can be solved independently. The velocity components in the seepage field are obtained from the solution of the flow equation, and then the velocity is substituted into the advection-dispersion equation as input. This method called decoupling method has high computational efficiency (Zheng et al., 2016). In this paper, the decoupling method was adopted to solve the problem of the flow velocity term in the advection-dispersion equation. According to the different influencing factors, different formulas for calculating the velocity term were given. The velocity term was substituted for the difference formula of the concentration, and the calculation results were expressed as the spatial distribution of chloride ion concentration in the seepage interval under this single influencing factor.

In the paper, the one-dimensional advection-dispersion equation was taken as the research object. It was considered that seawater and freshwater were miscible, regardless of source and sink terms, and the porosity $n$ is constant (Khakimov et al., 2017).

$$
\begin{gathered}
\frac{\partial c}{\partial t}=\frac{\partial}{\partial x}\left(D_{l} \frac{\partial c}{\partial x}\right)-\frac{\partial}{\partial x}\left(V_{x} c\right) \\
V_{x}=-k \frac{\partial h}{\partial x} \\
D_{l}=\alpha_{L}\left|V_{x}\right|
\end{gathered}
$$

In the above equations, $k$ is the hydraulic conductivity, unit $\mathrm{m} / \mathrm{s} ; D_{l}$ represents the longitudinal dispersivity coefficient, which is the physical quantity reflecting the dispersivity of soluble substances through the permeable media, unit $\mathrm{m}^{2} / \mathrm{s} ; a_{L}$ represents the longitudinal dispersivity, unit $\mathrm{m}$. 


\section{Explicit differentiation of differential equations}

In terms of one-dimensional advection-dispersion seawater intrusion model, there are two kinds of cases. Namely, the one case that seawater head is higher than freshwater head is defined as active SWI, and the other case that freshwater head is higher than seawater head is defined as passive SWI (Badaruddin et al., 2017). Under active SWI conditions, $h_{0}>h_{N}$, as shown in Figure 1, the direction of flow velocity is the same as the direction of salt migration as well as the direction of $\mathrm{X}$-axis. Furthermore, under passive SWI conditions, $h_{0}<h_{N}$, as shown in Figure 2, the direction of velocity is opposite to the direction of salt migration as well as the direction of the X-axis. The diffusion of salinity under constant flux, head and steady-state conditions was studied in this paper. Therefore it is assumed that the water head and flow velocity have reached a steady state before salinity diffusion.

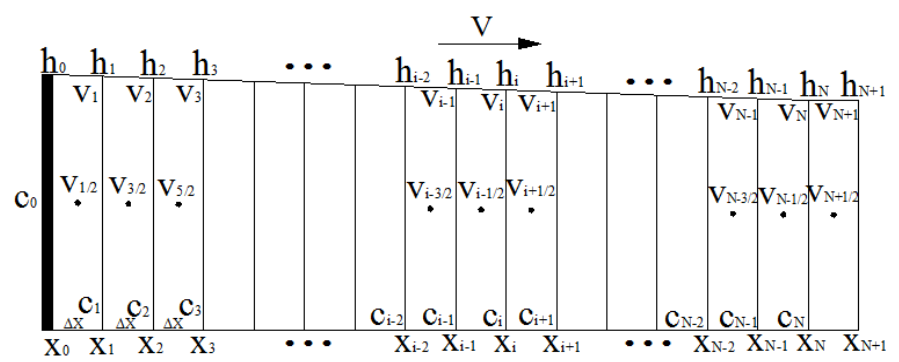

Figure 1. The seepage interval node division under active SWI conditions

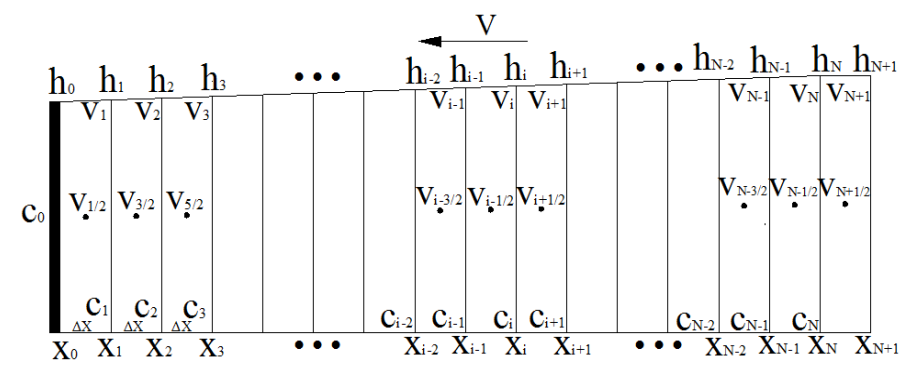

Figure 2. The seepage interval node division under passive SWI conditions

Under active SWI conditions, Equation 1 can be transformed into differential iterative formula as follows:

$$
\frac{c_{i}^{n+1}-c_{i}^{n}}{\Delta t}=\frac{\alpha_{L}\left[V_{i+\frac{1}{2}}\left(c_{i+1}^{n}-c_{i}^{n}\right)-V_{i-\frac{1}{2}}\left(c_{i}^{n}-c_{i-1}^{n}\right)\right]}{(\Delta x)^{2}}-\frac{V_{i} c_{i}^{n}-V_{i-1} c_{i-1}^{n}}{\Delta x} \quad(1 \leq i<N)
$$

When $i=N$, then $c_{N+1}^{n}=\beta c_{N}^{n}, V_{N+1 / 2}=V_{N}, \beta$ is the boundary attenuation factor, and its value is between $[0,1]$, reflecting the degree of attenuation of the concentration at the boundary. Therefore, Equation 1 can be transformed into Equation 5 as follows:

$$
\frac{c_{N}^{n+1}-c_{N}^{n}}{\Delta t}=\frac{\alpha_{L}\left[V_{N}(\beta-1) c_{N}^{n}-V_{N-\frac{1}{2}}\left(c_{N}^{n}-c_{N-1}^{n}\right)\right]}{(\Delta x)^{2}}-\frac{V_{N} c_{N}^{n}-V_{N-1} c_{N-1}^{n}}{\Delta x} \quad(i=N)
$$


Equations 4 and 5 can be transformed into standard difference formulas as follows:

$$
\left\{\begin{array}{l}
\Delta t /(\Delta x)^{2}=\lambda \\
c_{i}^{n+1}=\lambda \alpha_{L} V_{i+\frac{1}{2}} c_{i+1}^{n}+\left[1-\lambda\left(\alpha_{L} V_{i+\frac{1}{2}}+\alpha_{L} V_{i-\frac{1}{2}}+\Delta x V_{i}\right)\right] c_{i}^{n}+\lambda\left(\alpha_{L} V_{i-\frac{1}{2}}+\Delta x V_{i-1}\right) c_{i-1}^{n}(1 \leq i<N) \\
c_{N}^{n+1}=\left\{\left[1-\lambda\left[\alpha_{L}(1-\beta) V_{N}+\alpha_{L} V_{N-\frac{1}{2}}+\Delta x V_{N}\right]\right\} c_{N}^{n}+\lambda\left(\alpha_{L} V_{N-\frac{1}{2}}+\Delta x V_{N-1}\right) c_{N-1}^{n} \quad(i=N)\right.
\end{array}\right.
$$

The value of $\Delta x$ and $\Delta t$ should satisfy the corresponding convergence conditions. Under active SWI conditions, when the value of $\Delta x$ and $\Delta t$ should satisfy Equation 7 , the one-dimensional explicit difference schemes are absolutely convergent.

$$
1-\frac{\Delta t}{(\Delta x)^{2}}\left(\alpha_{L} V_{i+\frac{1}{2}}+\alpha_{L} V_{i-\frac{1}{2}}+\Delta x V_{i}\right) \geq 0
$$

$q$ stands for flux per unit width, whose calculation equation is shown in Equation 8, and its unit is $\mathrm{m}^{2} / \mathrm{s}(\mathrm{Qi}, 2016)$.

$$
q=\frac{k\left(h_{0}^{2}-h_{N}^{2}\right)}{2 L}
$$

Equation 9 is for calculating node water head height under active SWI conditions.

$$
h_{i}=\sqrt{h_{0}^{2}-i \frac{\left(h_{0}^{2}-h_{N}^{2}\right) \Delta x}{L}}
$$

Equations 10 and 11 are for calculating flow velocity under active SWI conditions.

$$
\begin{gathered}
V_{i}=\frac{q}{h_{i}}=\frac{k\left(h_{0}^{2}-h_{N}^{2}\right)}{2 L \sqrt{h_{0}^{2}-i \frac{\left(h_{0}^{2}-h_{N}^{2}\right) \Delta x}{L}}} \\
V_{i+\frac{1}{2}}=\frac{k\left(h_{i}-h_{i+1}\right)}{\Delta x}=\frac{k\left(\sqrt{h_{0}^{2}-i \frac{\left(h_{0}^{2}-h_{N}^{2}\right) \Delta x}{L}}-\sqrt{\left.h_{0}^{2}-(i+1) \frac{\left(h_{0}^{2}-h_{N}^{2}\right) \Delta x}{L}\right)}\right.}{\Delta x}
\end{gathered}
$$

Under passive SWI conditions, the calculated value of the velocity substituted is negative. Considering that $D_{l}$, the longitudinal dispersivity coefficient, is not affected by the forward or negative effect of the velocity, but only related to its size, Equation 1 can be transformed into Equation 12 as shown below to ensure that the coefficients of the velocity term in the explicit difference equation are forward.

$$
\frac{c_{i}^{n+1}-c_{i}^{n}}{\Delta t}=\frac{\alpha_{L}\left[V_{i+\frac{1}{2}}^{\prime}\left(c_{i+1}^{n}-c_{i}^{n}\right)-V_{i-\frac{1}{2}}^{\prime}\left(c_{i}^{n}-c_{i-1}^{n}\right)\right]}{(\Delta x)^{2}}+\frac{V_{i}^{\prime} c_{i}^{n}-V_{i-1}^{\prime} c_{i-1}^{n}}{\Delta x} \quad(1 \leq i<N)
$$

Similarly, Equation 12 can be transformed into standard difference formulas as follows: 


$$
\left\{\begin{array}{l}
\Delta t /(\Delta x)^{2}=\lambda \\
c_{i}^{n+1}=\lambda \alpha_{L} V_{i+\frac{1}{2}}^{\prime} c_{i+1}^{n}+\left[1-\lambda\left(\alpha_{L} V_{i+\frac{1}{2}}^{\prime}+\alpha_{L} V_{i-\frac{1}{2}}^{\prime}-\Delta x V_{i}^{\prime}\right)\right] c_{i}^{n}+\lambda\left(\alpha_{L} V_{i-\frac{1}{2}}^{\prime}-\Delta x V_{i-1}^{\prime}\right) c_{i-1}^{n}(1 \leq i<N) \\
c_{N}^{n+1}=\left\{\left[1-\lambda\left[\alpha_{L}(1-\beta) V_{N}^{\prime}+\alpha_{L} V_{N-\frac{1}{2}}^{\prime}-\Delta x V_{N}^{\prime}\right]\right\} c_{N}^{n}+\lambda\left(\alpha_{L} V_{N-\frac{1}{2}}^{\prime}-\Delta x V_{N-1}^{\prime}\right) c_{N-1}^{n} \quad(i=N)\right.
\end{array}\right.
$$

Under passive SWI conditions, its convergence conditions are shown as below:

$$
\left\{\begin{array}{c}
1-\frac{\Delta t}{(\Delta x)^{2}}\left(\alpha_{L} V_{i+\frac{1}{2}}^{\prime}+\alpha_{L} V_{i-\frac{1}{2}}^{\prime}-\Delta x V_{i}^{\prime}\right) \geq 0 \\
\alpha_{L} V_{i-\frac{1}{2}}^{\prime}-\Delta x V_{i-1}^{\prime} \geq 0
\end{array}\right.
$$

Equation 15 is for calculating node water head height under passive SWI conditions.

$$
h_{i}^{\prime}=\sqrt{h_{N}^{2}-(N-i) \frac{\left(h_{N}^{2}-h_{0}^{2}\right) \Delta x}{L}}
$$

Equations 16 and 17 are for calculating flow velocity under passive SWI conditions.

$$
\begin{gathered}
V_{i}^{\prime}=\frac{q}{h_{i}^{\prime}}=\frac{k\left(h_{N}^{2}-h_{0}^{2}\right)}{2 L \sqrt{h_{N}^{2}-(N-i) \frac{\left(h_{N}^{2}-h_{0}^{2}\right) \Delta x}{L}}} \\
V_{i+\frac{1}{2}}^{\prime}=\frac{k\left(h_{i}^{\prime}-h_{i+1}^{\prime}\right)}{\Delta x}=\frac{k\left(\sqrt{h_{N}^{2}-(N-i) \frac{\left(h_{N}^{2}-h_{0}^{2}\right) \Delta x}{L}}-\sqrt{\left.h_{0}^{2}-(N-i+1) \frac{\left(h_{N}^{2}-h_{0}^{2}\right) \Delta x}{L}\right)}\right.}{\Delta x}
\end{gathered}
$$

In order to study the effects of discharge variation, precipitation and evaporation on seawater intrusion, the flux is changed by keeping the hydraulic gradient constant and controlling the water head on both sides to increase at the same time.

The effects of rainfall and evaporation are considered to be uniform in the seepage field, and water exchange exists in each subzone. The position diagram of the influencing factors such as flow change, precipitation and evaporation was drawn, as shown in Figure 3.

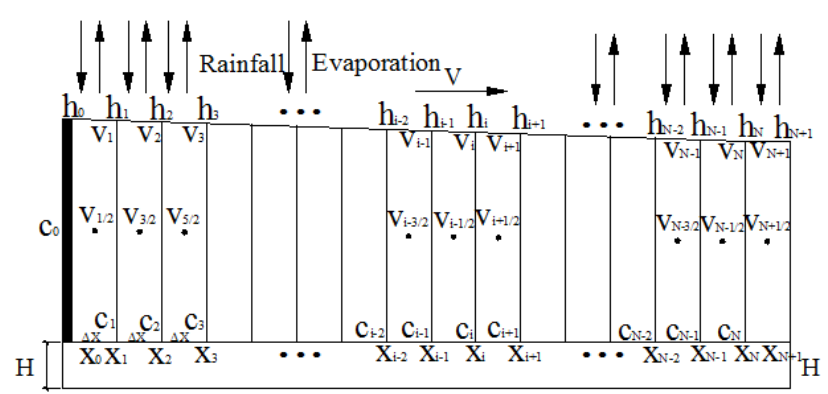

Figure 3. Influencing relationship of flux, rainfall and evaporation

As shown in Figure 3, $\mathrm{H}$ represents the numerical value of simultaneous rise of water head on both sides. If $H$ is positive, it means water head would increase $\mathrm{H}$ meters 
simultaneously on both sides. Conversely, negative $\mathrm{H}$ means decreasing. That is to say, the seawater head rises $H$ meters, at the same time, the fresh water level also rises $H$ meters. Meanwhile, the hydraulic gradient does not change. However, the flux changes with the change of $H$, which is a special flow quantity change, thus affecting the seepage velocity and the final concentration distribution. The formulas for calculating discharge, head and velocity can be seen in Equation 18.

$$
\left\{\begin{array}{l}
q_{H}=\frac{k\left[\left(h_{0}+H\right)^{2}-\left(h_{N}+H\right)^{2}\right]}{2 L} \\
h_{H_{i}}=\sqrt{h_{0}^{2}-i \frac{\left(h_{0}^{2}-h_{N}^{2}\right) \Delta x}{L}+H} \\
V_{H_{i}}=\frac{q_{H}}{h_{H}}, V_{H_{i+\frac{1}{2}}}=\frac{k\left(h_{H_{i}}-h_{H_{i+1}}\right)}{\Delta x}
\end{array}\right.
$$

As to rainfall and evaporation, the influencing factors on seawater intrusion, the annual average rainfall is represented by $R$, and the annual average evaporation is represented by $E$. The unit is $\mathrm{mm} / \mathrm{yr}$, and $1 \mathrm{~mm} / \mathrm{yr}=3.171 \times 10^{-11} \mathrm{~m} / \mathrm{s}$. Rainfall and evaporation are directly affected on the unit seepage length, and water exchange occurs in each spatial grid. Rainfall and evaporation will affect the flux in the seepage region. Thus, the flow velocity and salt diffusion are affected.

Under active SWI conditions, the equations of flux and flow velocity under the influence of the evaporation factor were shown below.

$$
\left\{\begin{array}{c}
q_{E_{i}}=\frac{k\left(h_{0}^{2}-h_{N}^{2}\right)}{2 L}-i E \\
V_{E_{i}}=\frac{q_{E_{i}}}{h_{i}}, V_{E_{i+\frac{1}{2}}}=\frac{k\left(h_{i}-h_{i+1}\right)}{\Delta x}
\end{array}\right.
$$

Under active SWI conditions, the equations of flux and flow velocity under the influence of the rainfall factor were shown below.

$$
\left\{\begin{array}{c}
q_{R_{i}}=\frac{k\left(h_{0}^{2}-h_{N}^{2}\right)}{2 L}+i R \\
V_{R_{i}}=\frac{q_{R_{i}}}{h_{i}}, V_{R_{i+\frac{1}{2}}}=\frac{k\left(h_{i}-h_{i+1}\right)}{\Delta x}
\end{array}\right.
$$

Under passive SWI conditions, the equations of flux and flow velocity are similar to those under active SWI conditions.

\section{Numerical examples}

It is assumed that there is a seepage tank, which is used to simulate seawater invasion. The length of the tank is $1.5 \mathrm{~m}$, the width is $0.1 \mathrm{~m}$, and $k=0.0001 \mathrm{~m} / \mathrm{s}, \alpha_{L}=0.2 \mathrm{~m}$. The left side is the boundary of constant head and concentration, the left side of seawater head is $0.5 \mathrm{~m}$, and the initial chloride ion 
concentration of the left side seawater is $18.000 \mathrm{mg} / \mathrm{L}$, which can be prepared by seawater salinometer measuring. The right side is the fixed head boundary, the fresh water head on the right side is $0.35 \mathrm{~m}$, and the chloride ion concentration is 0 . Under this condition, it is considered that there is no concentration decay at the right side boundary $\left(c_{N+1}^{n}=c_{N}^{n}\right)$, which is a case of active SWI, as shown in Figure 4. As to passive SWI, in the left side, the seawater head is $0.35 \mathrm{~m}$ and the initial chloride ion concentration is 0 ; and in the right side, the fresh water head is $0.5 \mathrm{~m}$ and the initial chloride ion concentration is $18.000 \mathrm{mg} / \mathrm{L}$, as shown in Figure 5. Besides, the physical photograph of the applied seepage tank is shown in Figure 6. Taking $\Delta x=0.03 \mathrm{~m}$, $\Delta t=100 \mathrm{~s}$ and $\beta=1$, the relationship will be calculated between the steady concentration and the diffusion distance of seawater salt in the seepage field after 6,000 iterations. The initial and boundary conditions are as shown below.

$$
\left\{\begin{array}{c}
\frac{\partial C}{\partial t}=\frac{\partial}{\partial x}\left(a_{L} \mid V_{x} \frac{\partial C}{\partial x}\right)-\frac{\partial}{\partial x}\left(V_{x} C\right) \quad(0<x<1.5, t>0) \\
\left.C\right|_{t=0}=0 \\
\left.C\right|_{x=0}=18000 \\
\left.\frac{\partial V}{\partial x}\right|_{x=1.5}=0
\end{array}\right.
$$

While the finite difference method to calculate the above examples are used, under the condition of convergence, after 6000 iterations, the concentration of the node position basically does not change with the increase of iterations. Meanwhile, the calculation results represent the chloride ion concentration under steady state, then the degree of seawater intrusion in the region will be judged.

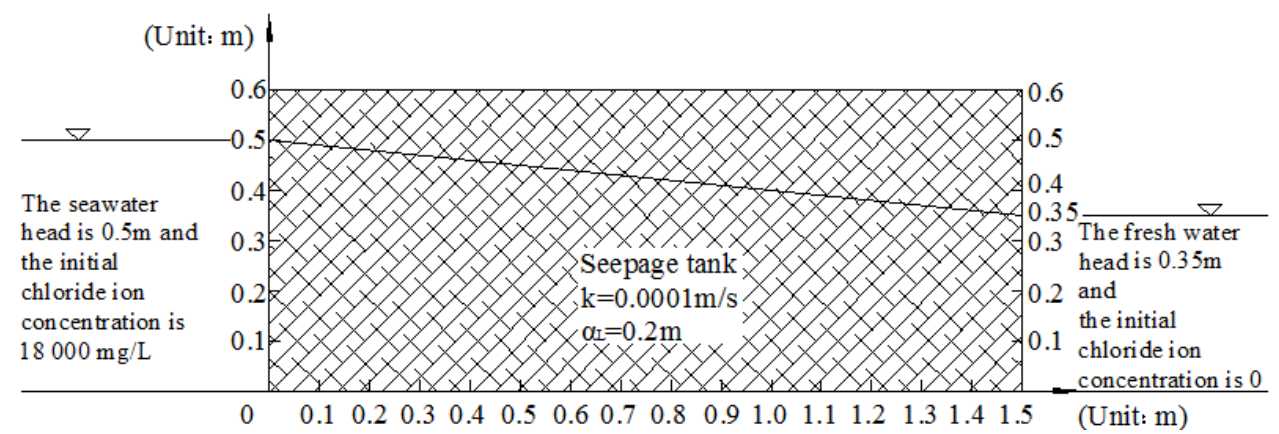

Figure 4. The seepage tank size and boundary conditions under active SWI conditions

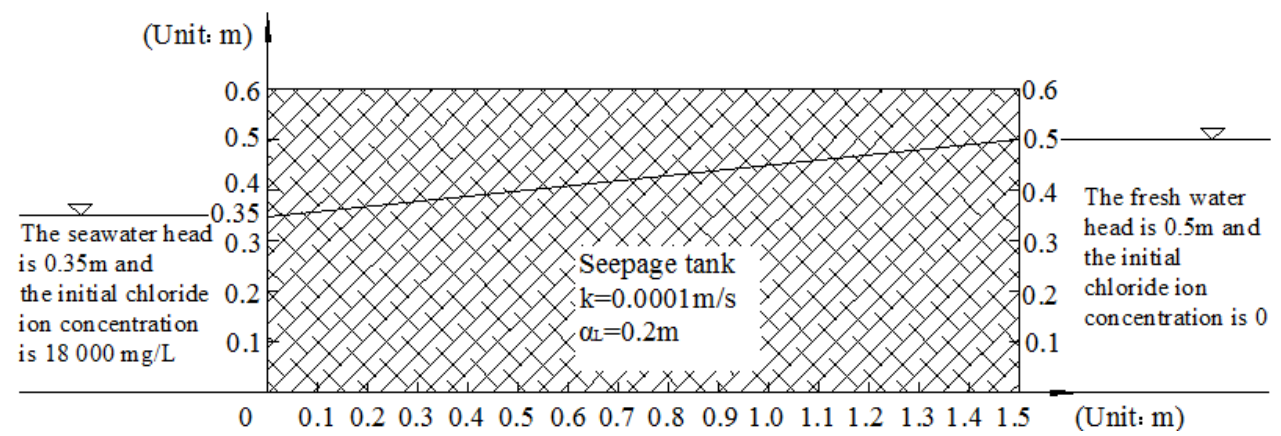

Figure 5. The seepage tank size and boundary conditions under passive SWI conditions 


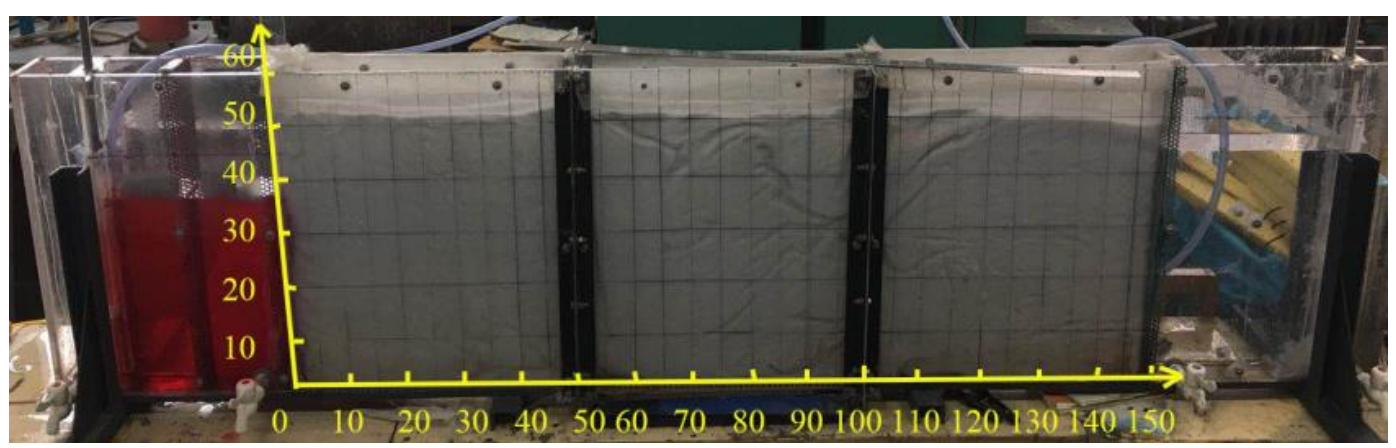

Figure 6. The physical photograph of the applied seepage tank (unit, $\mathrm{cm}$ )

\section{Result discussion}

The effects of six hydraulic factors on seawater intrusion were explored in this paper, namely, hydraulic conductivity, longitudinal dispersivity, hydraulic gradient, flux, evaporation and rainfall. Besides, the initial and boundary conditions utilized for calculation and simulation were based on the contents described in the numerical examples above. In this paper, the finite difference method was utilized to solve onedimensional advection-dispersion equation. Meanwhile, the control variates method was adopted to explore the influence of a single factor on seawater invasion. After iteration calculation, the distribution of chloride ion concentration in the seepage field was compared under steady state.

\section{Hydraulic conductivity}

Hydraulic conductivity $k$, which is defined as the unit flux under the unit hydraulic gradient, represents the difficulty of fluid passing through the pore framework. The hydraulic conductivity can affect the flow velocity of seepage flow and the salt migration. In order to study the influence of hydraulic conductivity on salt migration, five different numerical values of hydraulic conductivity were formulated in this paper, namely, $k=0.00005 \mathrm{~m} / \mathrm{s}, 0.0001 \mathrm{~m} / \mathrm{s}, 0.00015 \mathrm{~m} / \mathrm{s}, 0.0002 \mathrm{~m} / \mathrm{s}$ and $0.00025 \mathrm{~m} / \mathrm{s}$. Hydraulic conductivity of sandy soil is related to many factors, such as material composition, particle size, shape, gradation and compactness of soils (Liu et al., 2017), and it can be monitored nondestructively and rapidly in real time by the resistivity measurement (Yuan et al., 2019).

Keeping other conditions unchanged, considering the forward and reverse seepage type conditions, the influence of hydraulic conductivity on seawater intrusion and its final state was analyzed, as shown in Figure 7.

According to the calculated results, the concentration of the final steady state rises with the increase of the hydraulic conductivity, but its growth rate is small. In the steady seepage field, the hydraulic conductivity is the time that affects the salinity to reach the final steady state. The larger the hydraulic conductivity is, the smaller the time to reach the steady state will be. Moreover, the hydraulic conductivity is forwardly correlated with the degree of seawater invasion.

\section{Longitudinal dispersivity}

Longitudinal dispersivity is related to the properties of porous media, unit $\mathrm{m}$, describing the degree of the dispersion in the migrating direction of flow, which is one 
of the most important parameters representing the dispersion feature of aquifer (Cheng, 2002), and it can be compute by the dispersive-area (DA) method and the linear graphic (LG) method (Zhang et al., 2010). Therefore, five different numerical values of longitudinal dispersivity were formulated in this paper, namely, $\alpha_{L}=0.1 \mathrm{~m}, 0.2 \mathrm{~m}$, $0.3 \mathrm{~m}, 0.4 \mathrm{~m}$ and $0.5 \mathrm{~m}$. Keeping other conditions unchanged, considering the forward and reverse seepage type conditions, the influence of longitudinal dispersivity on seawater intrusion and its final state was analyzed, as shown in Figure 8.

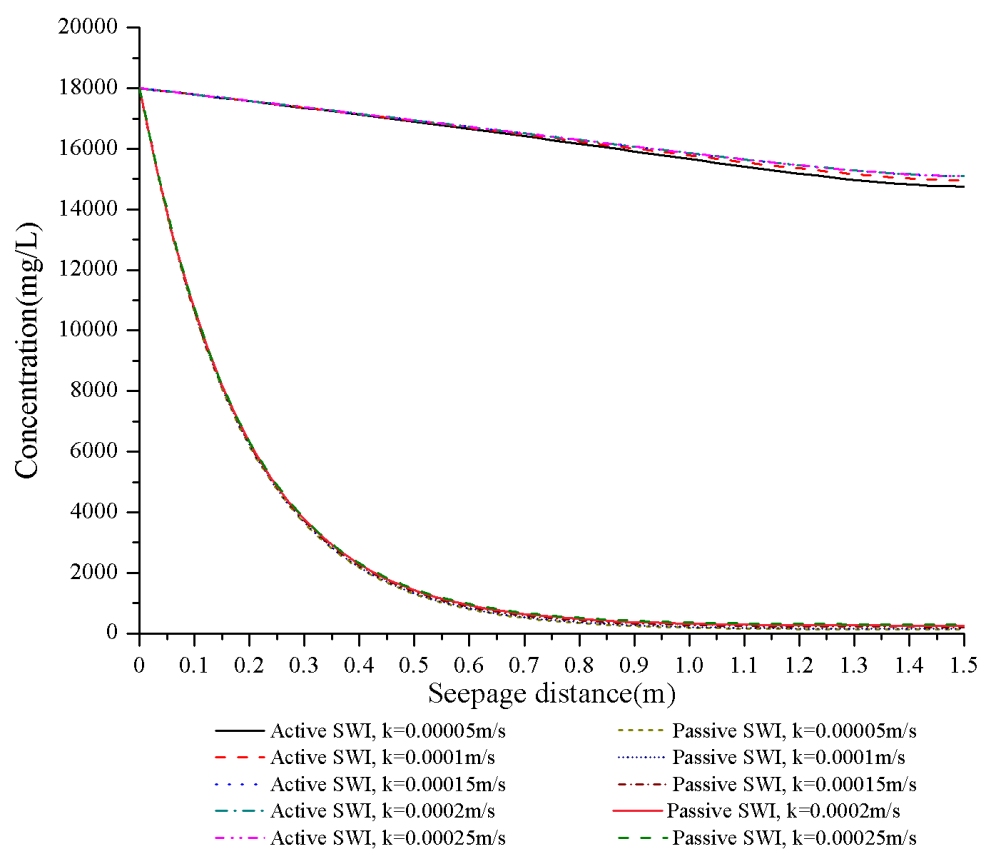

Figure 7. The concentration distribution map under the changing hydraulic conductivity

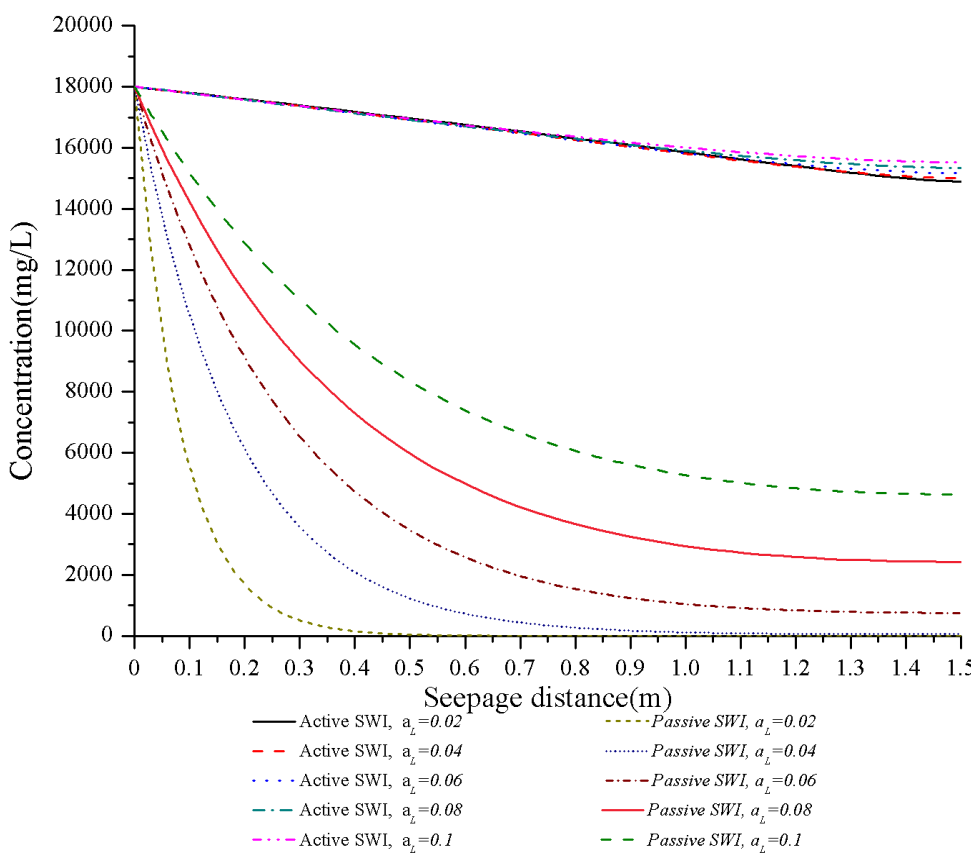

Figure 8. The concentration distribution map under the changing longitudinal dispersivity 
According to the calculated results, when the longitudinal dispersivity increases in the test condition, the concentration under steady state also grows, indicating that the longitudinal dispersivity is forwardly correlated with seawater intrusion. From Figure 8 it is found that the longitudinal dispersivity has a great influence on the degree of seawater intrusion under both steady state and passive SWI conditions, and this phenomenon is particularly evident under passive SWI conditions but inapparent under active SWI conditions. The greater the longitudinal dispersivity is, the severer the seawater invasion will be under both active and passive SWI conditions.

\section{Hydraulic gradient}

Hydraulic gradient, $J$, refers to the loss of head per unit distance of flow, $J=\left|h_{0}-h_{N}\right| / L$. Five different numerical values of hydraulic gradient were formulated in this paper, namely, $J=0.1 \mathrm{~m}, 0.2 \mathrm{~m}, 0.3 \mathrm{~m}, 0.4 \mathrm{~m}$ and $0.5 \mathrm{~m}$. Keeping other conditions unchanged, considering the forward and reverse seepage type conditions, the influence of hydraulic gradient on seawater intrusion and its final state was analyzed, as shown in Figure 9.

According to the calculation results, under the condition of active SWI, with the increase of the numerical value of hydraulic gradient, the concentration of chloride ion shows an increasing trend in the seepage field. However, under passive SWI conditions, with the increase of the numerical value of hydraulic gradient, the concentration of chloride ion decreases in the seepage field. That is to say, when the groundwater head increases, it will slow down the degree of seawater intrusion. On the contrary, when the head is reduced, the degree of seawater intrusion will be aggravated. In addition, this phenomenon is particularly evident under active and passive SWI conditions. Finally, the results of numerical simulation are the same as the achievements by the field measurements of Zhang et al. (2015) and also accord with the numerical experiments of Werner (2017).

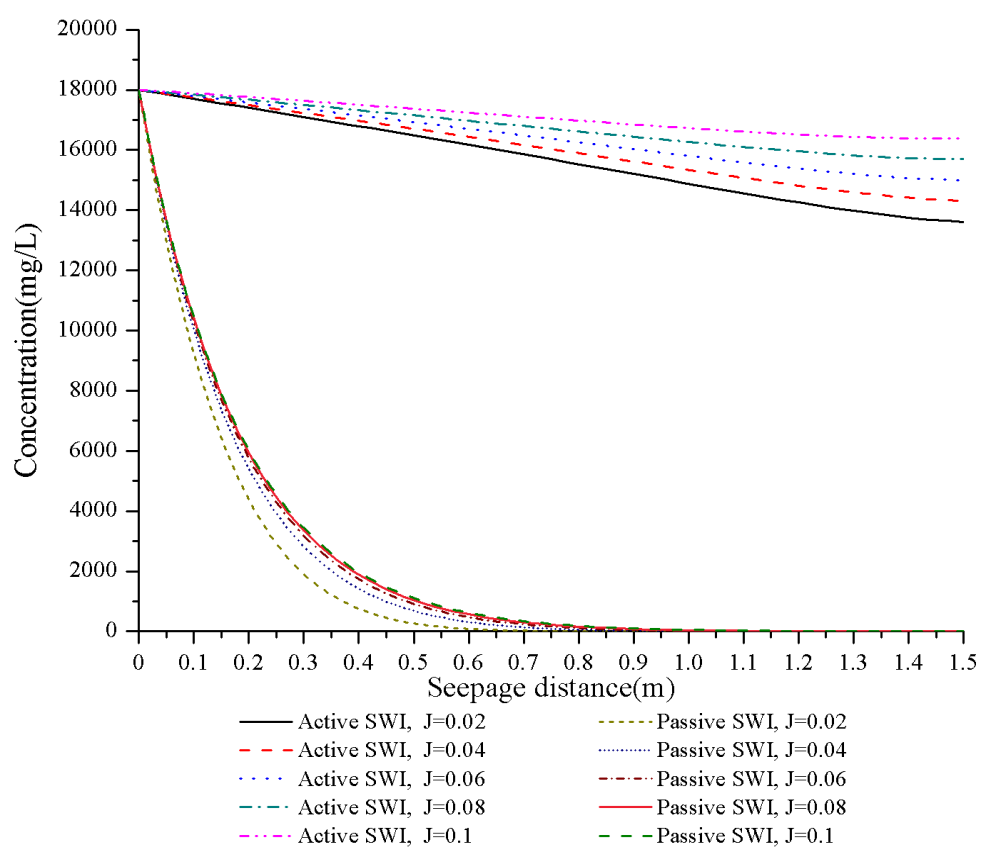

Figure 9. The concentration distribution map under the changing hydraulic gradient 


\section{Flux}

With the influence of flux, it was proposed that under the condition of constant hydraulic gradient, the water heads on the left and right sides should be raised or lowered at the same time, so as to make the flux increase or decrease while the hydraulic gradient remains unchanged. In order to explore the distribution of salt migration and chloride ion concentration under steady-state in different flow states, five working conditions were proposed. They were water head on both sides dropping by $0.1 \mathrm{~m}$ and by $0.05 \mathrm{~m}$, respectively, water head remaining unchanged and water head on both sides rising by $0.05 \mathrm{~m}$ and by $0.1 \mathrm{~m}$. Keeping other conditions unchanged, considering under the forward and reverse seepage types, the influence of flux on seawater intrusion under steady state was analyzed, as shown in Figure 10.

According to the calculation results, under active SWI conditions, the concentration of the final steady state increases with the increase of the flux. Although the final concentration also rises under passive SWI conditions, it was also slightly in terms of the extent of growth. Therefore, the flux was forwardly correlated with the degree of seawater intrusion.

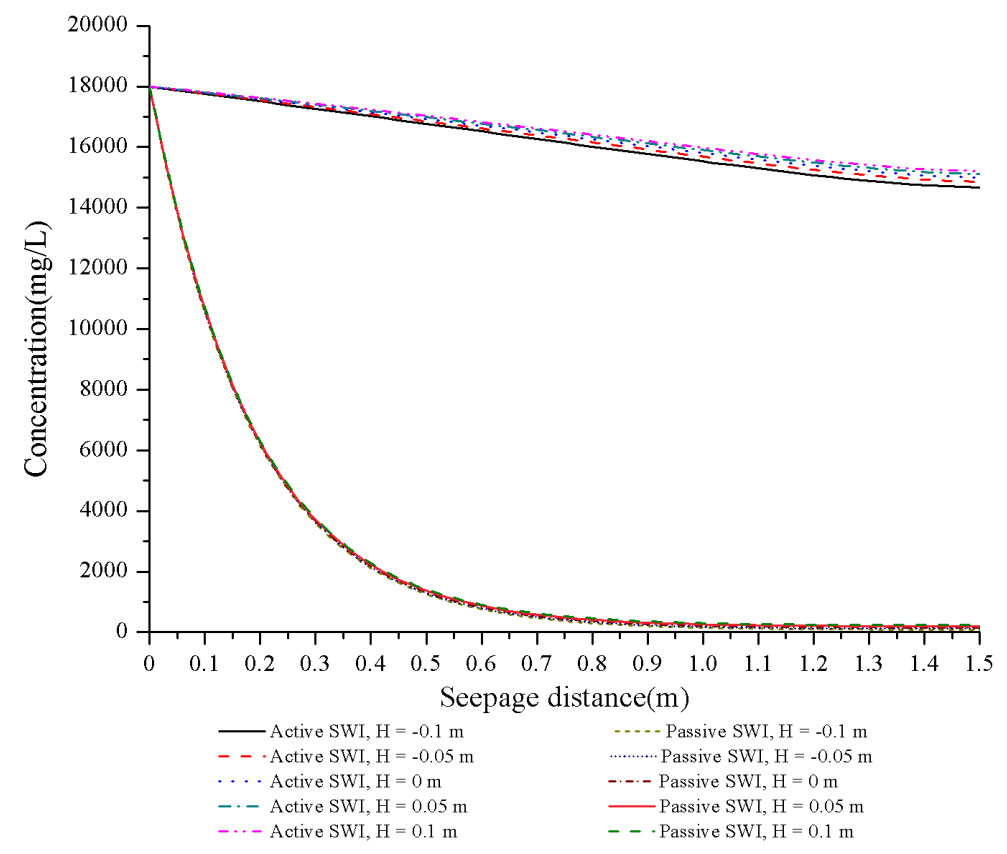

Figure 10. The concentration distribution map under the changing flux

\section{Annual average evaporation}

Evaporation refers to the amount of water that evaporates into the air over a certain period of time, which is usually expressed in millimeters of the thickness of the evaporated water layer, and it can be measured by the evaporimeter. In extremely arid areas, the average annual evaporation can reach more than $3000 \mathrm{~mm} / \mathrm{yr}$, for example, in western Ordos Plateau, China (Sun et al., 2017). Therefore, five different numerical values of annual average evaporation were formulated in this paper, namely, $1000 \mathrm{~mm} / \mathrm{yr}, 2000 \mathrm{~mm} / \mathrm{yr}, 3000 \mathrm{~mm} / \mathrm{yr}$ and $4000 \mathrm{~mm} / \mathrm{yr}, 5000 \mathrm{~mm} / \mathrm{yr}$. Keeping other conditions unchanged, considering the forward and reverse seepage type conditions, the 
influence of annual average evaporation on seawater intrusion and its final state was analyzed, as shown in Figure 11.

According to the calculated results, under active SWI conditions, with the increase of the average annual evaporation, the concentration of the final steady state increases with a small range. Although the final concentration also grows under passive SWI conditions, it was also insignificantly in terms of the extent of growth. Therefore, the annual average evaporation was forwardly correlated with the degree of seawater intrusion. That is to say, the greater the degree of evaporation is, the severer the seawater invasion will be.

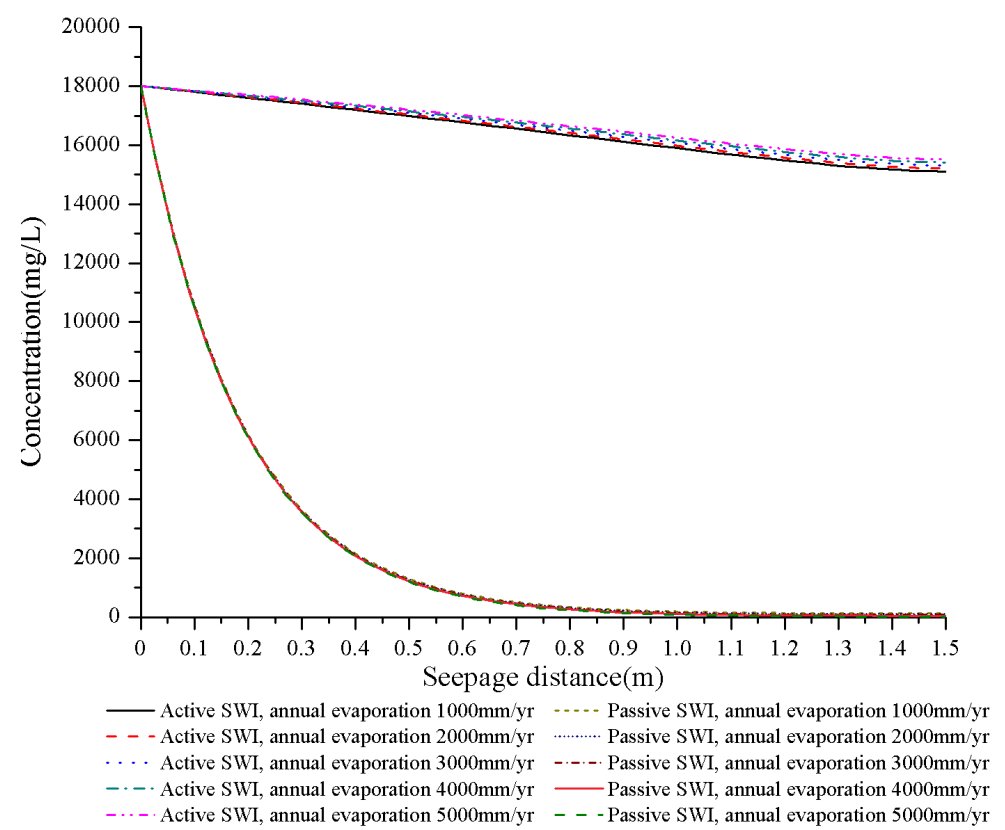

Figure 11. The concentration distribution map under the changing annual average evaporation

\section{Annual average rainfall}

Rainfall refers to the depth of the water layer falling to the ground in a certain period of time, which is generally measured by the rainfall measuring cylinder. The average annual rainfall in China is about $630 \mathrm{~mm} / \mathrm{yr}$, but in some extremely humid areas, the average annual rainfall can reach more than $10.000 \mathrm{~mm} / \mathrm{yr}$. Therefore, five different numerical values of annual average rainfall were formulated in this paper, namely, $0 \mathrm{~mm} / \mathrm{yr}, 1000 \mathrm{~mm} / \mathrm{yr}, 2000 \mathrm{~mm} / \mathrm{yr}, 3000 \mathrm{~mm} / \mathrm{yr}$ and $4000 \mathrm{~mm} / \mathrm{yr}$. Keeping other conditions unchanged, considering the forward and reverse seepage type conditions, the influence of annual average rainfall on seawater intrusion and its final state was analyzed, as shown in Figure 12.

According to the calculated results, under active SWI conditions, with the increase of average annual rainfall, the concentration in the final steady state decreases with a small extent. Under passive SWI conditions, the concentration also reduces slightly. Therefore, the rainfall is negatively correlated with the seawater invasion degree, and rainfall can slow down the degree of seawater invasion. Finally, the results of numerical simulation accord with the achievements of Qing et al. (2013). 


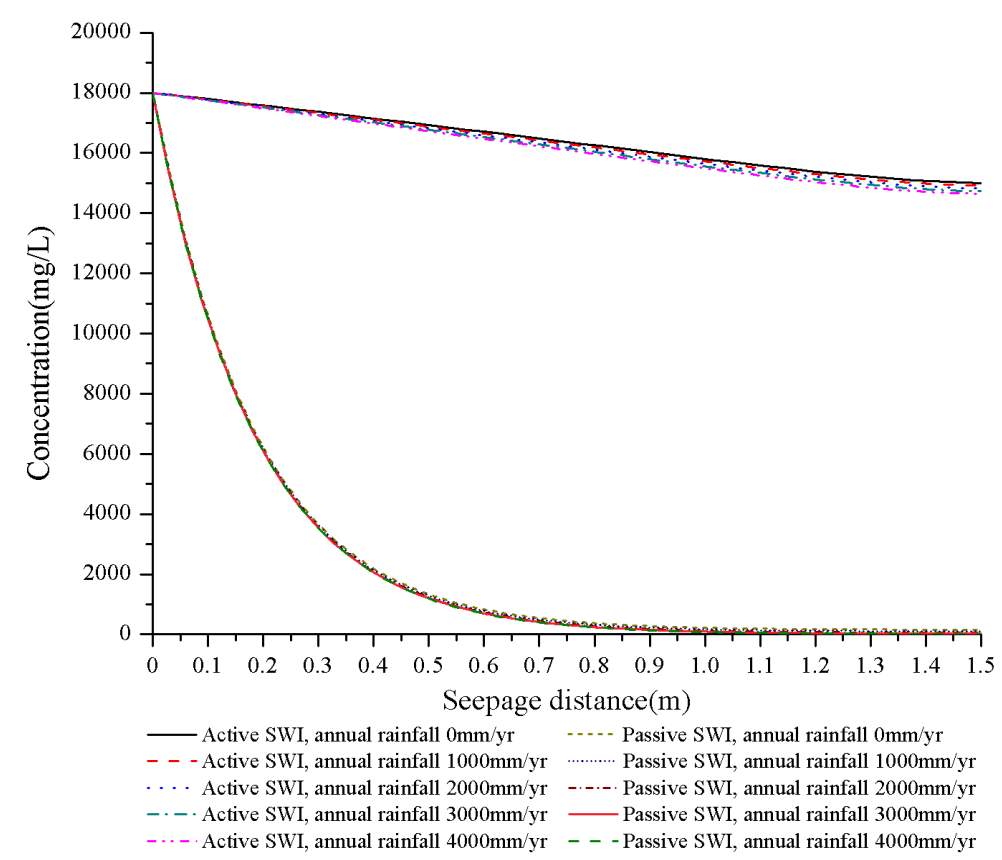

Figure 12. The concentration distribution map under the changing annual average rainfall

\section{Conclusion}

(1) In this paper, this numerical simulation method of solving one-dimensional advection-dispersion equation by explicit finite difference method can obtain distribution of chloride ion concentration at spatial nodes under steady state. Moreover, this method can be utilized to explore the influence of different factors on the degree of seawater intrusion, and the results and achievements are efficient, robust, reliable and consistent with the actual situation, which can explain the phenomenon of seawater intrusion. In addition, the numerical method for solving the one-dimensional advectiondispersion equation by the explicit finite difference method is correct and worthy of being used for reference. Compared with the one-dimensional advection-dispersion equation, the two-dimensional equation can more accurately explain the salt transport process under different SWI conditions. Therefore, it should be worth studying with the two-dimensional Taylor's theorem and finite difference method.

(2) It can be seen from the results of numerical simulation that, with the increase of the numerical value of hydraulic conductivity, longitudinal dispersivity, flux and evaporation capacity, the degree of seawater invasion will be aggravated, but the rainfall can play a role in mitigating seawater intrusion. Besides, under active SWI conditions, the growth of the numerical value of hydraulic gradient will aggravate seawater intrusion, while under passive SWI conditions, it will slow down the degree of invasion. As for the reason, the six factors can affect the distribution of groundwater velocity field (the size and direction of groundwater velocity), thus affecting seawater intrusion. In addition, some other factors should be worth studying, for example, pumping positions and pumping quantity.

(3) It can be seen from the results of numerical simulation that under both active and passive SWI conditions, the above six hydraulic factors have a significant influence, to a certain extent, on the seawater intrusion under steady state. Furthermore, the effect of hydraulic gradient on seawater intrusion is greatly obvious under both active and 
passive SWI conditions. Additionally, it is found that the longitudinal dispersivity has a great influence on the degree of seawater intrusion under both steady state and passive SWI conditions, and this phenomenon is particularly evident under passive SWI conditions but inapparent under active SWI conditions.

Acknowledgements. This paper can be completed thanks to the help of Professor Shaoxiong Zhang, who gave this paper some professional guidance. At the same time, I am also grateful for the help of Professor Gaoyue Rong on the writing of this paper. This paper was supported by the Natural Science Fund of Hebei Province (Grant No. E2017210094).

\section{REFERENCES}

[1] Adel, Z., Amira, M., Lahcen, Z., Jamila, T. (2014): Understanding groundwater chemistry using multivariate statistics techniques to the study of contamination in the Korba unconfined aquifer system of Cap-Bon (North-east of Tunisia). - Journal of African Earth Sciences 3: 1-15.

[2] Badaruddin, S., Werner, A. D., Morgan, L. K. (2017): Characteristics of active seawater intrusion. - Journal of Hydrology 551: 632-647.

[3] Chen, G. Q. (2013): Mechanisms Underlying of Seawater Intrusion and Evaluation of Early Warning Systems in the Laizhou Bay Area. - East China Normal University, Shanghai.

[4] Cheng, J. M. (2002): Analysis on field scale effect of dispersivity in consideration of relative reliability level of data. - Hydraulic Journal 2: 90-94.

[5] Cui, Z., Chen, G. Q., Xu, X. Y. (2015): Mechanism and assessment of seawater intrusion in the Northern Changshan Island. - Marine Environmental Science 34: 930-936.

[6] Emna, B., Moez, B., Ikram, J., Nabila, A., Jalila, M., Hafedh, K., Salem, B. (2018): Hydrochemical and statistical studies of the groundwater salinization combined with MODPATH numerical model: case of the Sfax coastal aquifer, Southeast Tunisia. Arabian Journal of Geosciences 11(4).

[7] Khakimov, E., Chung, S. Y., Senapathi, V., Elzain, H. E., Son, J. (2017): The characteristics of hydrogeological parameters of unconsolidated sediments in the Nakdong River delta of Busan City, Korea. - Journal of Soil and Groundwater Environment 22(3): 27-41.

[8] Li, G., Chen, C. X. (1996): The development and trend in researches of saltwater intrusion. - Earth Science Frontiers 3: 161-169.

[9] Liu, X. H. (2007): Experimental Analysis of Seawater Intrusion Speed in Sands. - Dalian Maritime University, Dalian.

[10] Liu, Y., Zhao, Y. R. (2017): Experimental study on influence factors of permeability coefficients of sand soil. - Journal of Jiangsu Construction 185: 88-91.

[11] Mao, Y. T. (2014): Technical Regulations for Sea Water Invasion Monitoring (Trial Implementation). - Department of Environmental Protection, State Oceanic Administration, Qingdao.

[12] Miao, Q. (2013): Effects of Precipitation and Tide on Seawater Intrusion in the Laizhou Bay. - First Institute of Oceanography, State Oceanic Administration, Qingdao.

[13] Nie, J., Zhao, Q. S. (2002): Research status and development trend on the seawater intrusion mathematical model. - Journal of Anshan Normal University 4: 16-18.

[14] Purnaditya, N. P., Soeryantono, H., Marthanty, D. R. (2018): Proposing mathematical model for seawater intrusion phenomena in the coastal aquifer. - MATEC Web of Conferences 197: 10003.

[15] Qi, Q. L. (2016): Hydraulics. - China Railway Press, Beijing. 
[16] Savovic, S., Djordjevich, A. (2012): Finite difference solution of the one-dimensional advection- diffusion equation with variable coefficients in semi-infinite media. International Journal of Heat and Mass Transfer 55(15-16): 4291-4294.

[17] Sun, J., Wang, Q. M., Liu, J. (2018): Change of the potential evapotranspiration in western Ordos Plateau: taking Etoke County as an example. - Journal of Irrigation and Drainage 11: 84-90.

[18] Werner, A. D. (2017): On the classification of seawater intrusion. - Journal of Hydrology 11: 619-631.

[19] Xue, Y. Q., Xie, C. H., Wu, J. C. (1992): Seawater invasion. - Hydrogeology and Engineering Geology 19: 29-34.

[20] Yang, S. L. (2016): Boundary value methods for solving the fractional advectiondispersion equation. - Journal of Computational Mathematics of Colleges and Universities 38: 350-356.

[21] Yu, C., Wang, H., Fang, D. F. (2018): Semi-analytical solution to one-dimensional advective-dispersive- reactive transport equation using homotopy analysis method. Journal of Hydrology 565: 422-428.

[22] Yuan, Z. H., Wang, Y. R., Peng, C., Mei, L., Wang, L. Y. (2019): Measurement of sands permeability coefficient based on electrical resistivity. - Journal of Yangzhou University (Natural Science Edition) 3: 60-65.

[23] Zhang, J., Wang, M. Y., Zhang, L. (2010): Suitability of numerical simulation for two computations of longitudinal dispersivity in heterogeneous media. - Journal of East China University of Science and Technology (Natural Science Edition) 6: 796-800.

[24] Zhang, W. J., Chen, X., Tan, H. B., Zhang, Y. F., Cao, J. F. (2015): Geochemical and isotopic data for restricting seawater intrusion and groundwater circulation in a series of typical volcanic islands in the South China Sea. - Marine Pollution Bulletin 93(1-2): 153162.

[25] Zhang, Y. H. (2015): Application of the change groundwater level in analysing seawater intrusion. - Marine Environmental Science 34: 788-791.

[26] Zhao, Z. W., Zhao, J., Xin, P., Hua, G. F., Jin, G. Q. (2018): Uncertainty and sensitivity analyses of the simulated seawater-freshwater mixing zones in steady-state coastal aquifers. - China Ocean Engineering 4: 489-502.

[27] Zheng, C. M., Bennett, G. D. (2016): Applied Contaminant Transport Modelling. $2^{\text {nd }}$ Ed. - Higher Education Press, Beijing. 